\title{
Use of Aptamers to deliver therapeutic genetic sequences in muscle
}

\section{LEONIDAS A. PHYLACTOU}




\section{Muscular Dystrophy}

-Group of muscle diseases

- Inherited

-Muscle weakness and wasting

- Duchenne Muscular Dystrophy, Becker Muscular Dystrophy, Myotonic Dystrophy

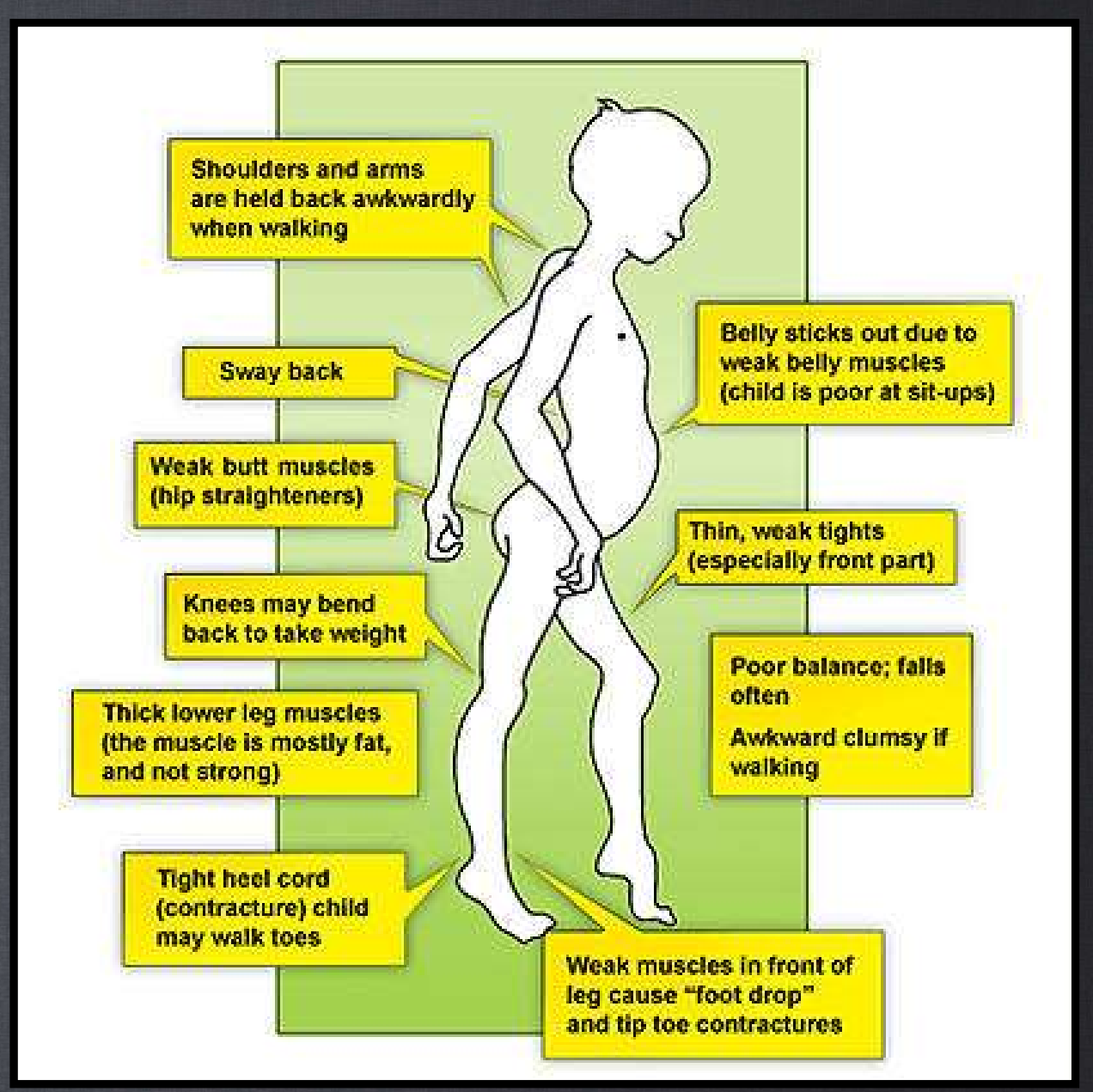




\section{MYOTONIC Dystrophy}

- Autosomal dominant

- Most common neuromuscular disease in adults

- Muscle weakness and wasting

- Other symptoms may include cataracts, intellectual disability, and heart conduction problems

- Type 1 (DM1) and type 2 (DM2)

- Anticipation

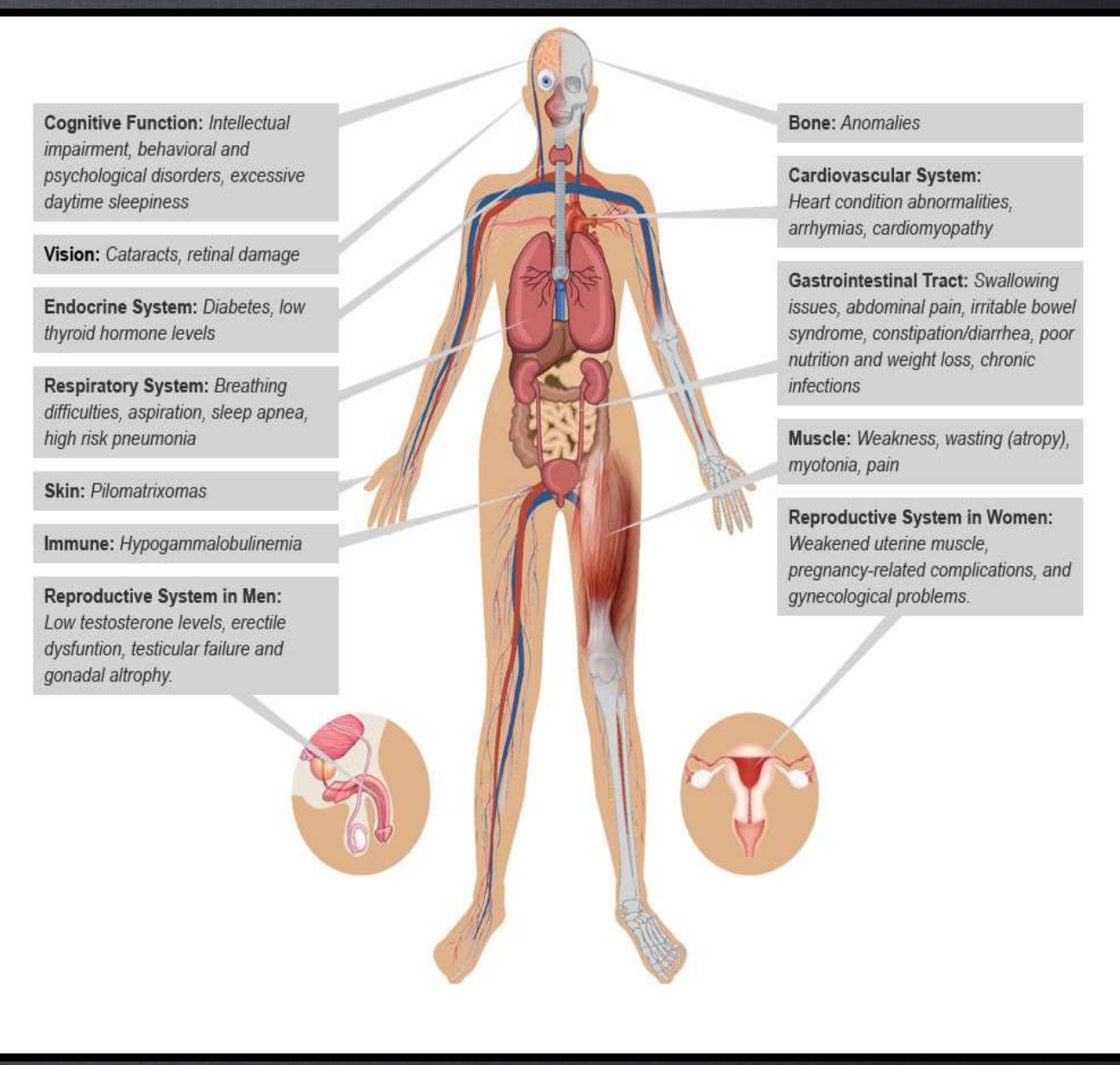

Thornton, C. A. (2014). "Myotonic dystrophy." Neurologic clinics 32(3): 705-719, viii. 


\section{DM1 Pathogenesis}

Nuclear retention of CUG ${ }^{\exp -M B N L 1}$ foci

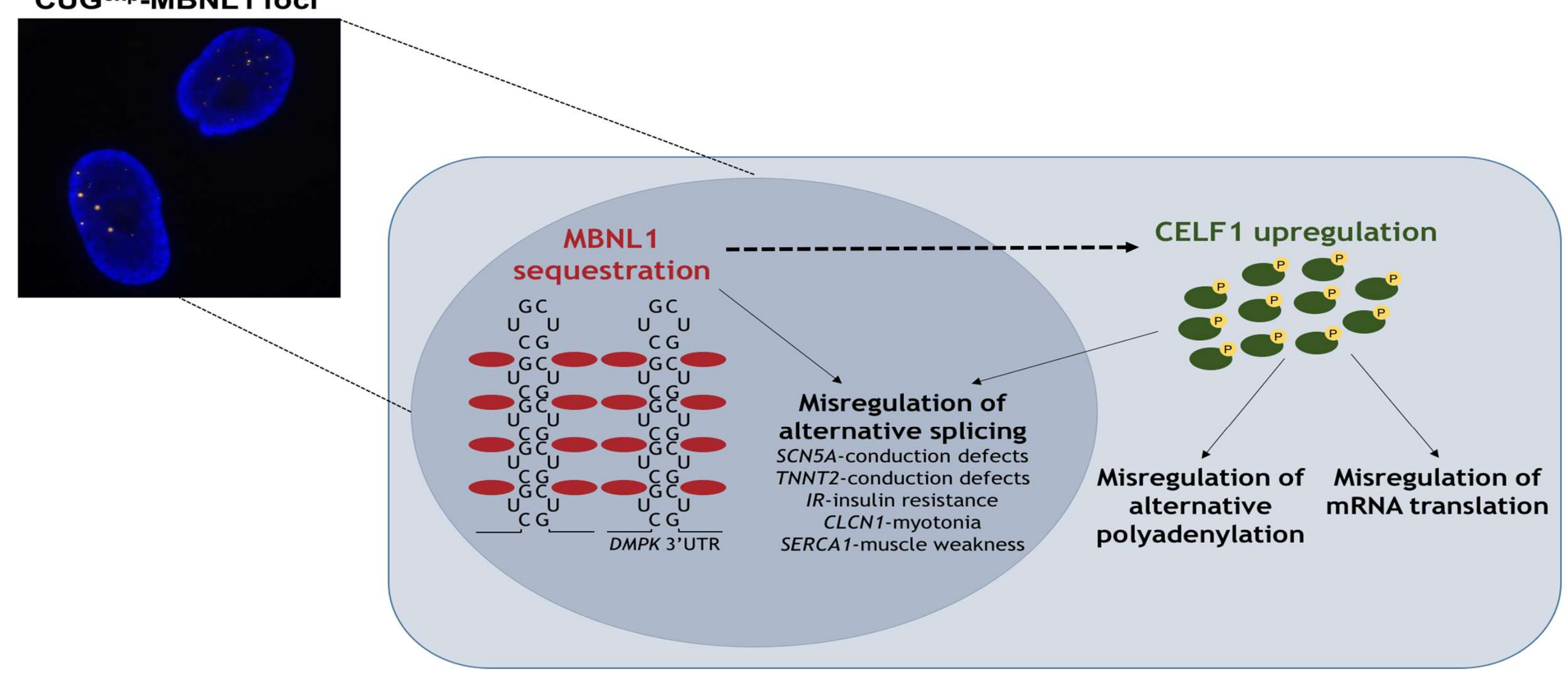

Thornton, C. A. (2014). "Myotonic dystrophy." Neurologic clinics 32(3): 705-719, viii. 


\section{ANTISENSE OLIGONUCLEOTIDES AGAINST MYOTONIC DYSTROPHY}

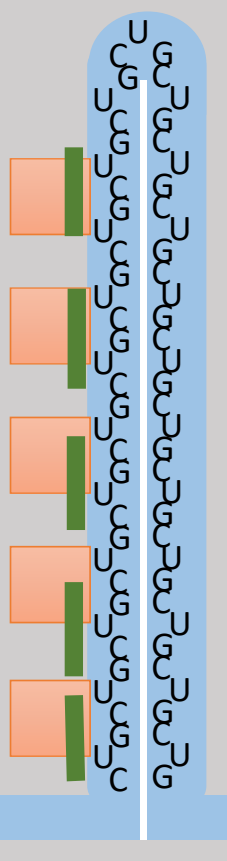




\section{Duchenne muscular Dystrophy}

- X-linked

- Most common form of muscular dystrophy

- 1 in 3,500 newborn boys

- Progressive muscle weakness and cardiomyopathy

- Ultimately die from cardiac or respiratory complications before their third decade of life

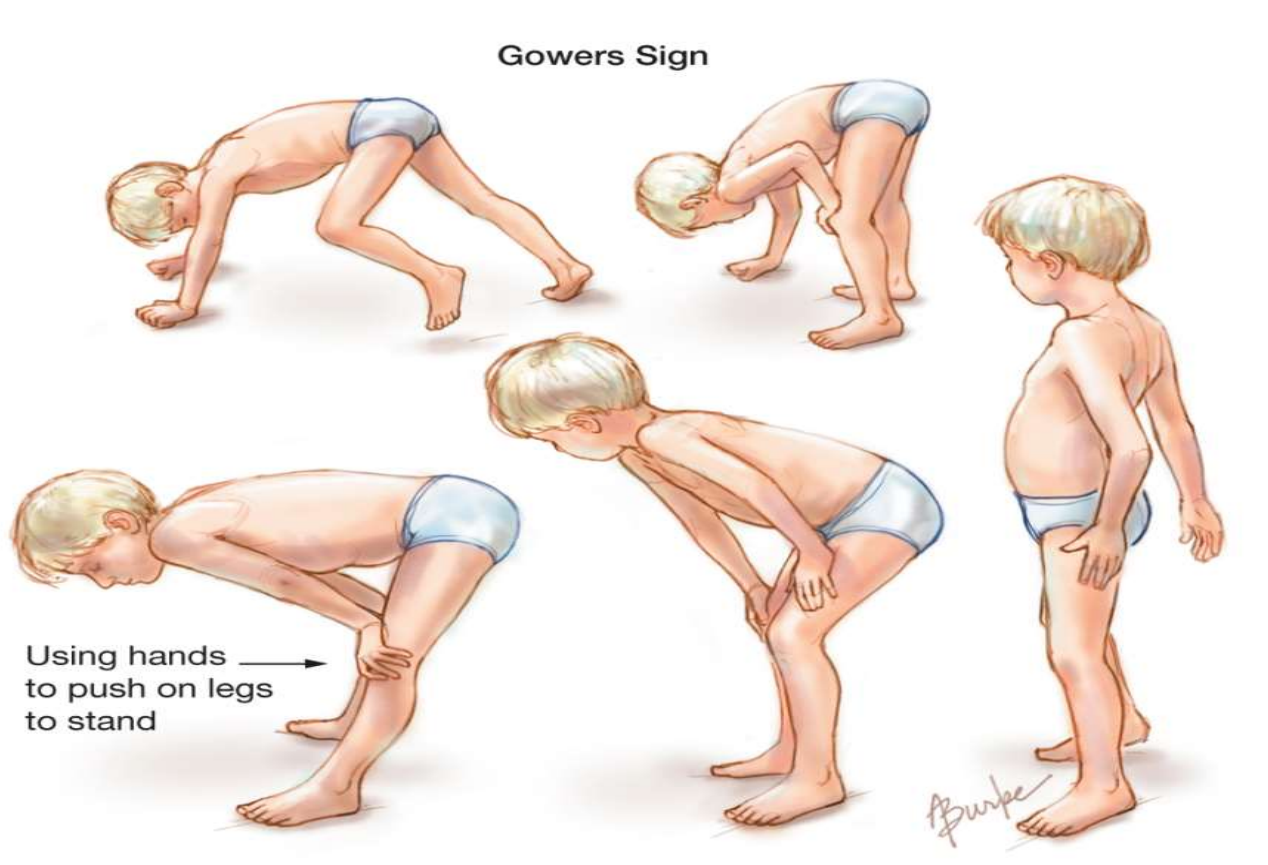

Chamberlain, J. R. and J. S. Chamberlain (2017). Molecular Therapy 25(5): 1125-1131 


\section{DMD gene}

- Dystrophin DMD gene is the largest known human gene (2.4 Mb), containing 79 exons

- Nonsense or frame-shift mutations

- Hotspots: deletions between exons 4555 and duplications between exons 2-10

- Thus these mutations lead to loss of dystrophin expression in the muscle fibres

- 1 in 3 cases is caused from a de novo mutation

A Normal

$\begin{array}{lcc}\longrightarrow \text { Translation start } & \text { Translation stop } \\ & * 79\end{array}$

Dystrophin transcript is translated into dystrophin protein

B Duchenne muscular dystrophy

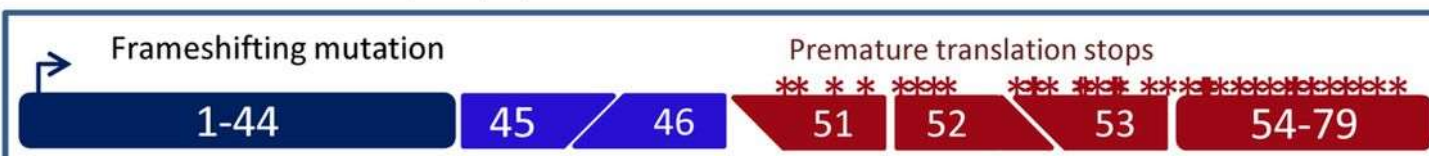

Incoorporation aberrant amino acids after deletion and generally premature truncation of translation

$\gg$ Nonsense mutation

Premature truncation of protein translation (reading frame is not affected) 


\section{DMD protein}

The dystrophin associated glycoprotein complex (DGC)

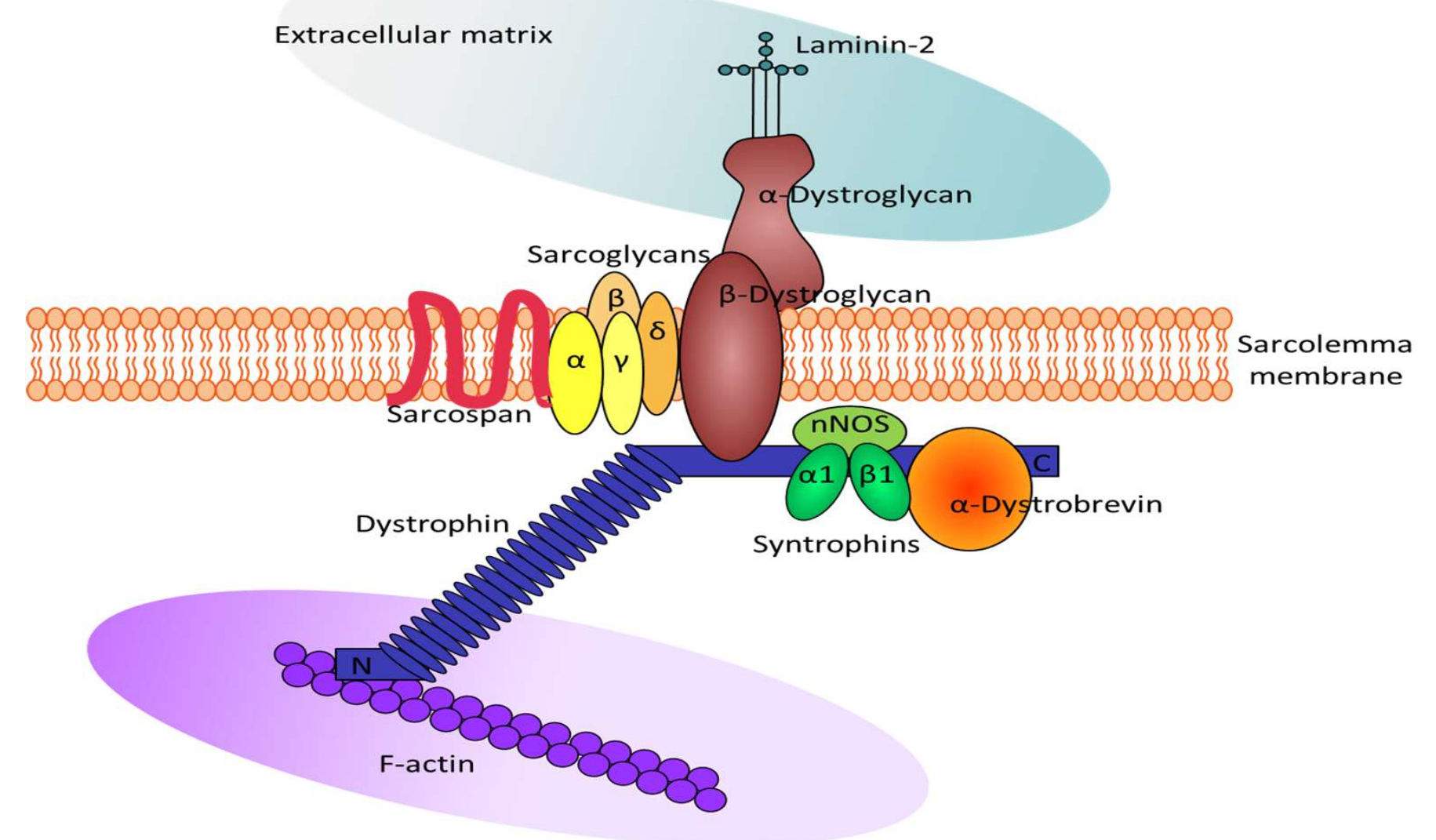




\section{DMD protein}

The dystrophin associated glycoprotein complex (DGC)
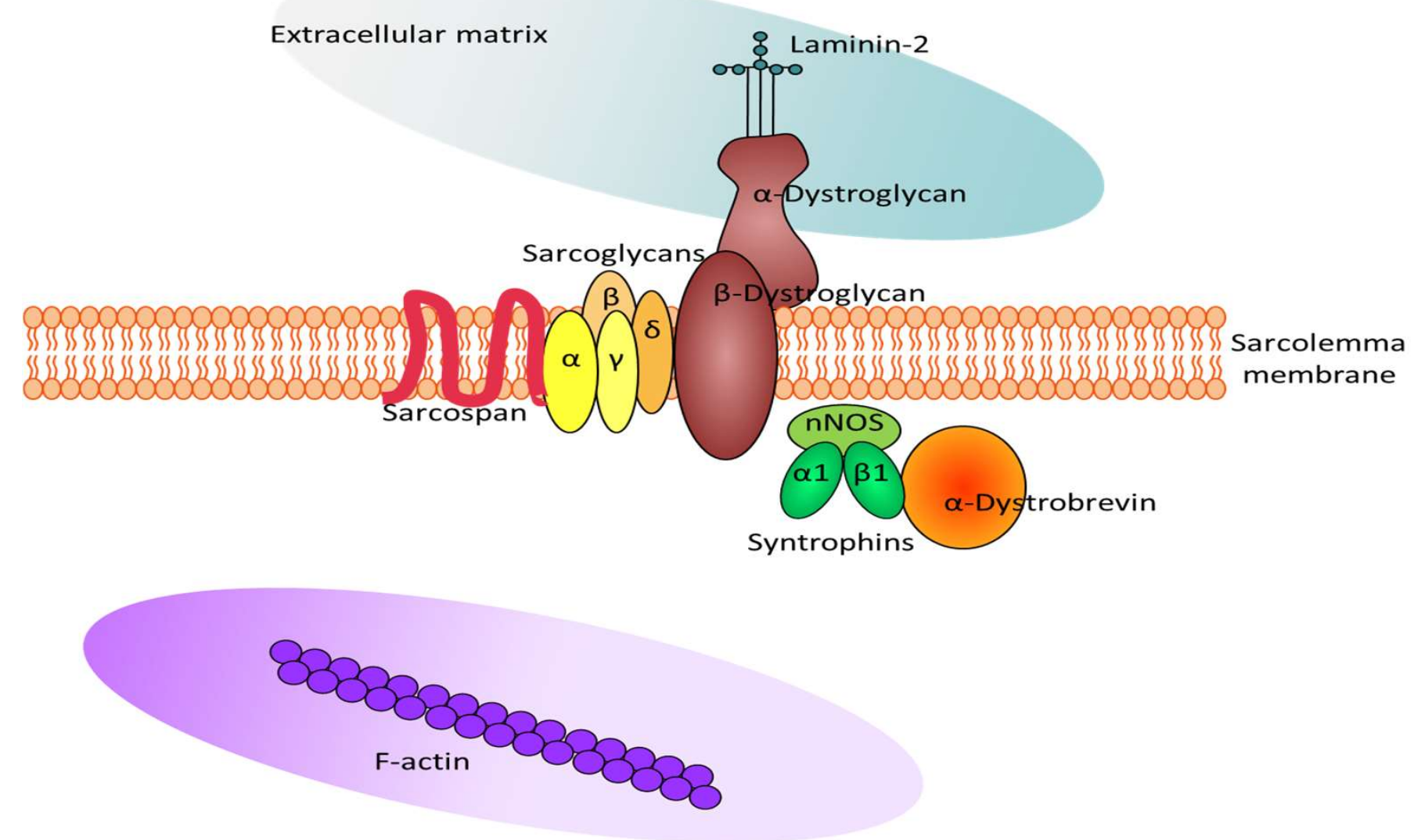


\section{Potential Therapeutic Treatment}

Exon skipping in the DMD $(m d x)$ mouse model

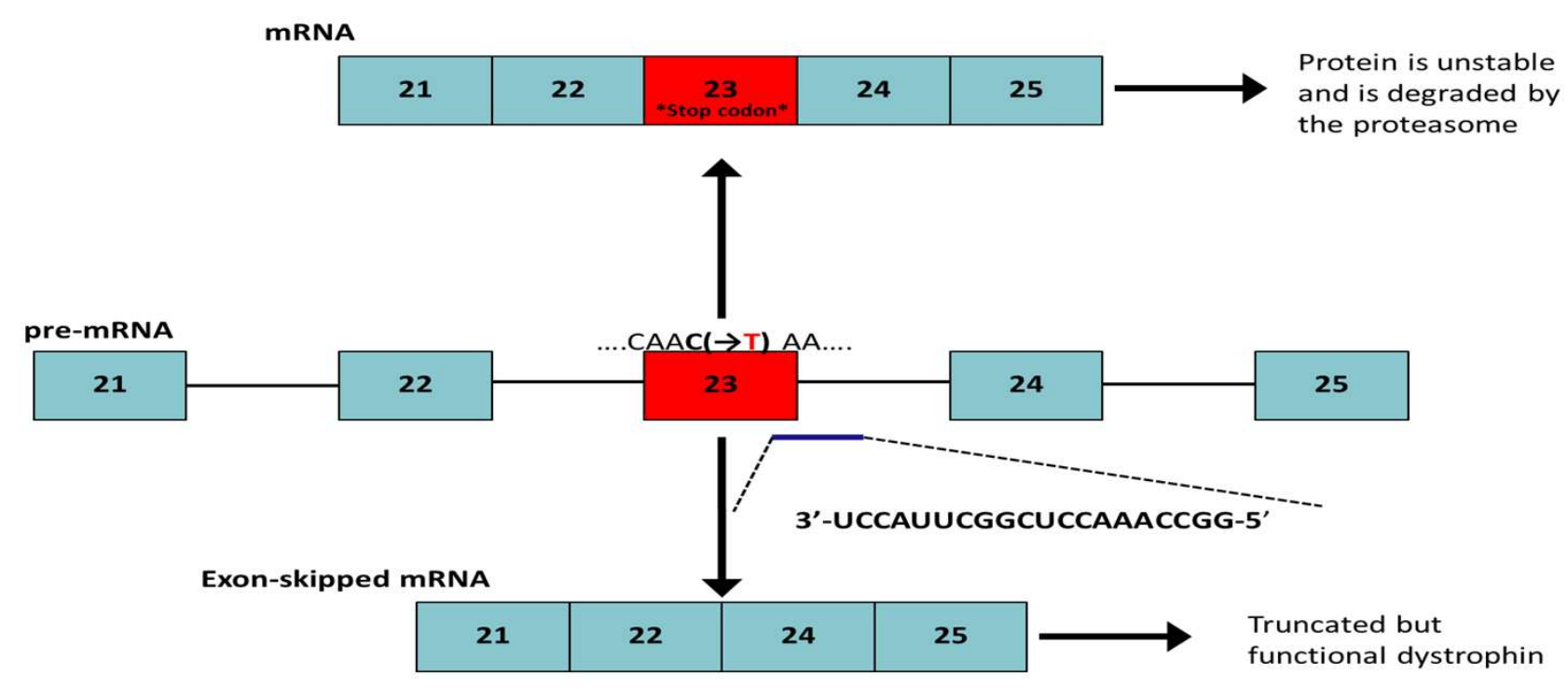

$83 \%$ of all DMD mutations could be treated by exon skipping 


\section{Delivery of AON in muscle}




\section{Aptamers}

- Synthetic nucleic acid molecules designed to bind with high specificity and affinity to a selected target.

- Fold into unique three-dimensional structures.

- Systematic Evolution of Ligands by Exponential Enrichment (SELEX).

$\checkmark$ "Survival of the fittest".

$\checkmark$ Has been modified in different ways for a number of applications.

$\checkmark$ Selective targeting of cells for the delivery of therapeutic molecules: siRNAs, miRNAs, chemotherapeutics and toxins.

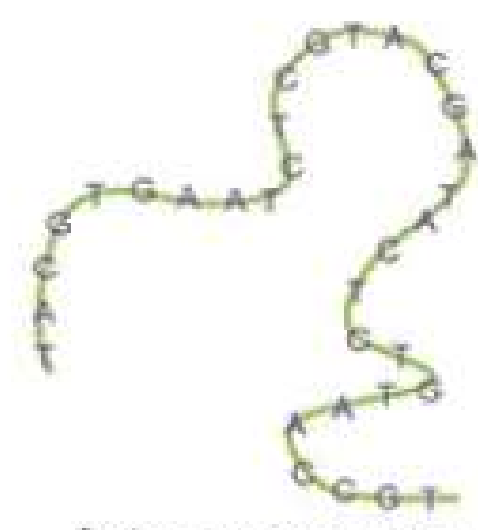

Aptamer sequence

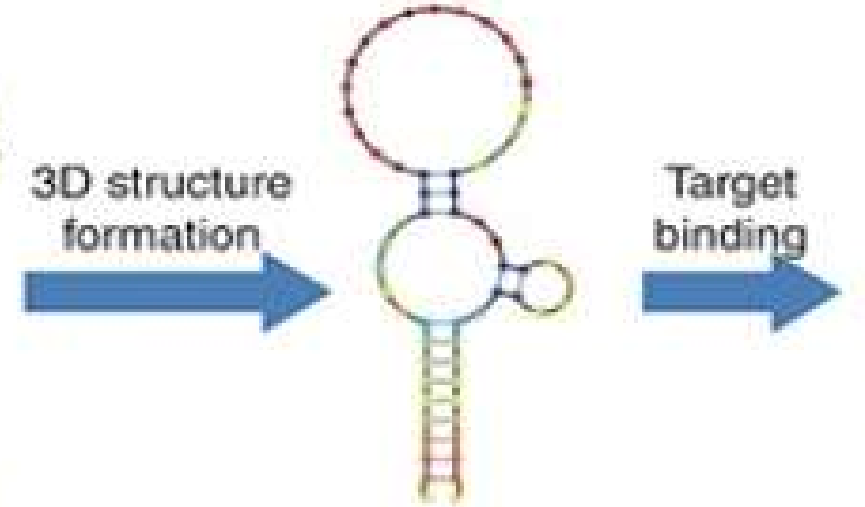

Functional aptamer

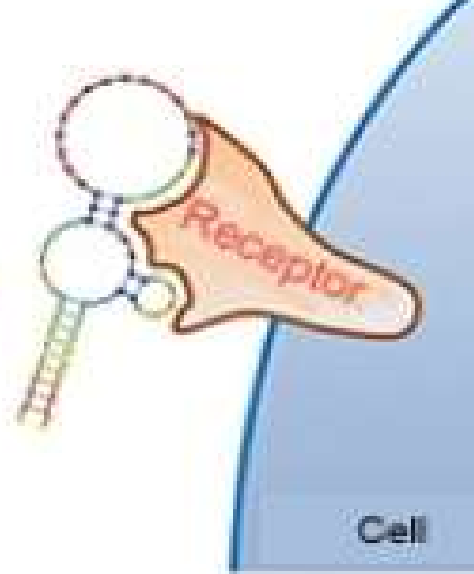




$$
\begin{gathered}
\text { Aptamer } \\
\text { Delivery of } \\
\text { AON in muscle }
\end{gathered}
$$




\section{Cell-Internalizing SELEX for skeletal muscle RNA aptamers}

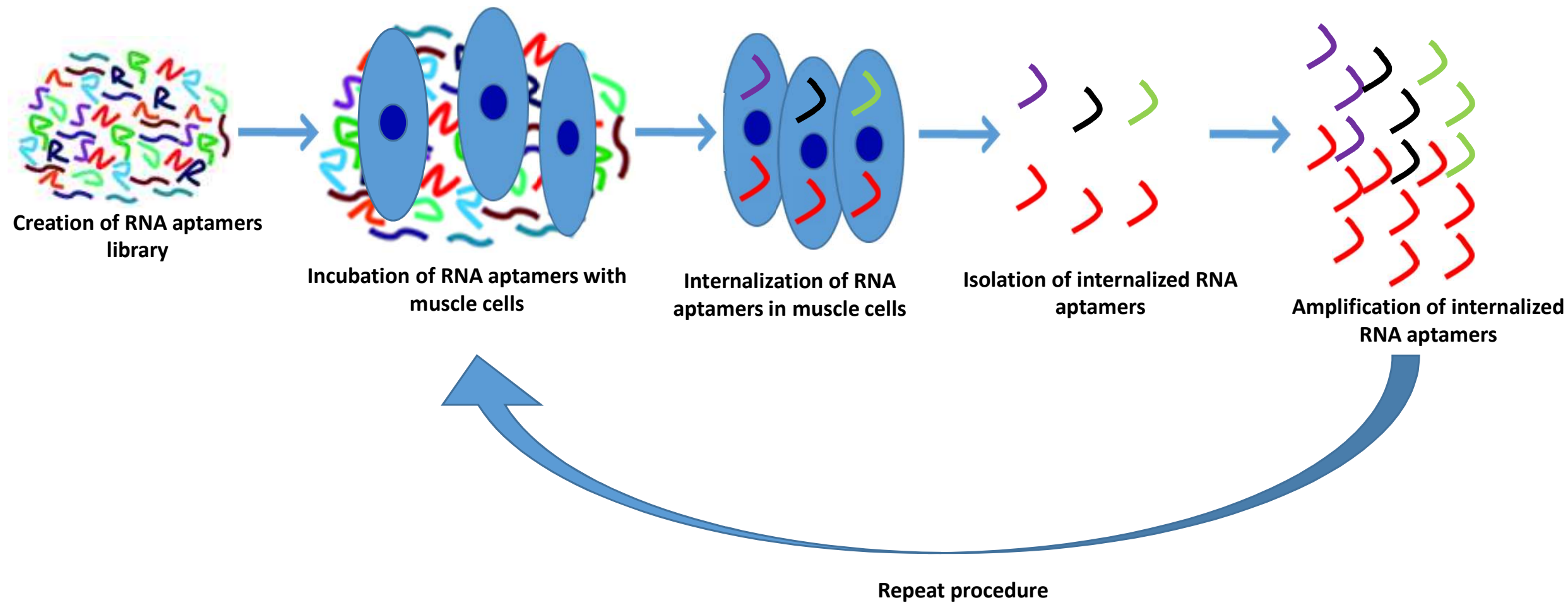

Philippou et. al. 2017 Molecular Therapy Nucleic Acids 
Fluorescein labelled RNA aptamers pool (round 15) + DAPI

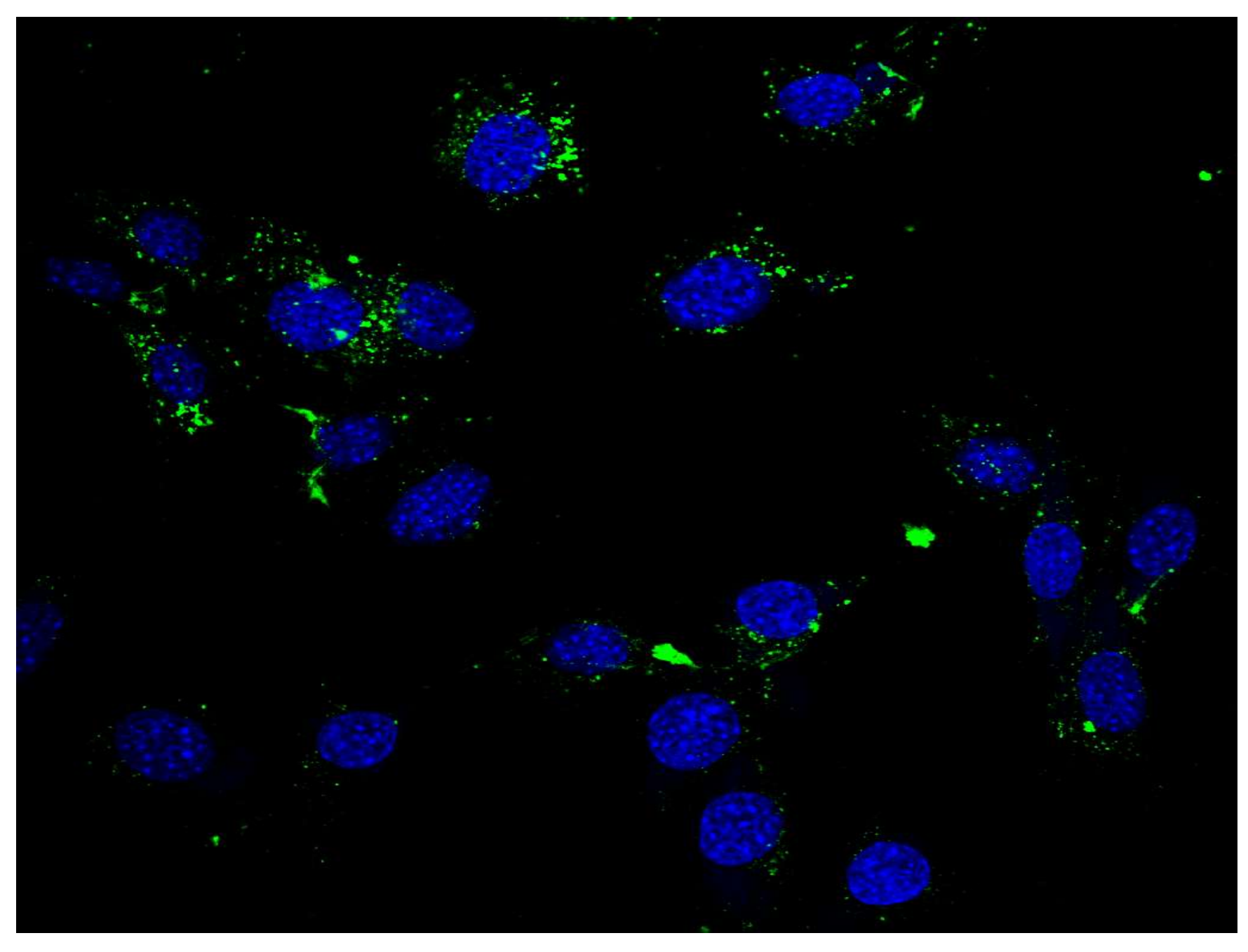




\section{Aptamer convergence and alignment}
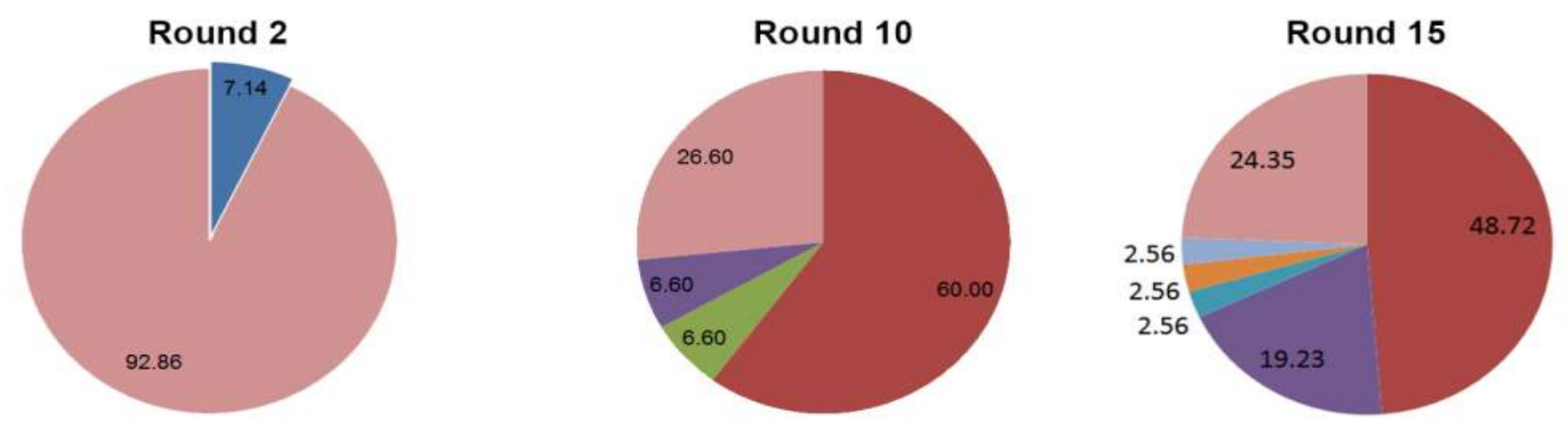

a Family 1

a Family 2

- \% Family 3

a Family 4

- \% Family 5

- \% Family 6

- \% Family 7

m \% Random

Family 1 5'-GGGAAGAGAAGGACATATGAT GGgGGgGAAGTGAGTACAGGAGgGCGCGAGTGTGCTGTAG TTGACTAGTACATGACCACTTGA-3'

$\Rightarrow$ Family 2 5'-GgGAAGAGAAGGACATATGAT CAGgAGCCGAGAACCGGTTGGTGGGTAATCCTGTTAGCGC TTGACTAGTACATGACCACTTGA-3' Family 3 5'-GGGAAGAGAAGGACATATGAT CCGGTTTGGCTGAGCGTCGGCGCTCTGGAAGATGGCGTGG TTGACTAGTACATGACCACTTGA-3'

7 Family 4 5'-GGGAAGAGAAGGACATATGAT GTGGGTGGGAGTTGGTAGGGCGATGGGGAGAGGTGGCTGG TTGACTAGTACATGACCACTTGA-3' Family 5 5'-GGGAAGAGAAGGACATATGAT CTATGGTGCGATAAAGGAGGCATCGCAATCGGTGGTGTGG TTGACTAGTACATGACCACTTGA-3' Family 6 5'-GGGAAGAGAAGGACATATGAT GGGTAGGTTGGTAAGGTGGGGGGAGGAGGCAGTGTGGCAG TTGACTAGTACATGACCACTTGA-3' Family 7 5'-GGGAAGAGAAGGACATATGAT TAGGGTGGCAGGTTAGGAGGGGAGGTAGGAGTGGGTGTGG TTGACTAGTACATGACCACTTGA-3'

$\Rightarrow$ Random 5'-GGGAAGAGAAGGACATATGAT N N N N N N N N N N N N N N N N N N N N N N N N N N N N N N N TTGACTAGTACATGACCACTTGA-3' 


\section{Internalization and cellular localization of}

A01B RNA aptamer in vitro

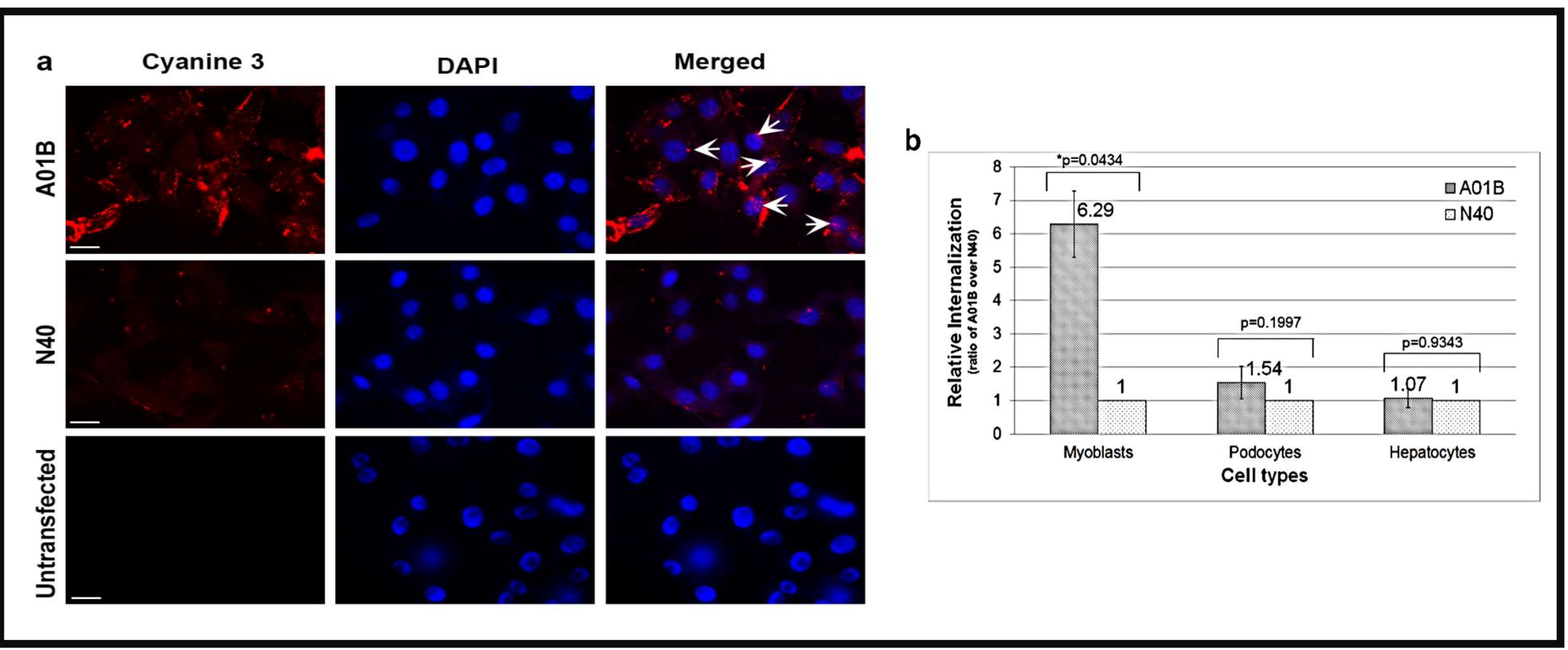




\section{A01B RNA aptamer was found free from early endosomal compartments}

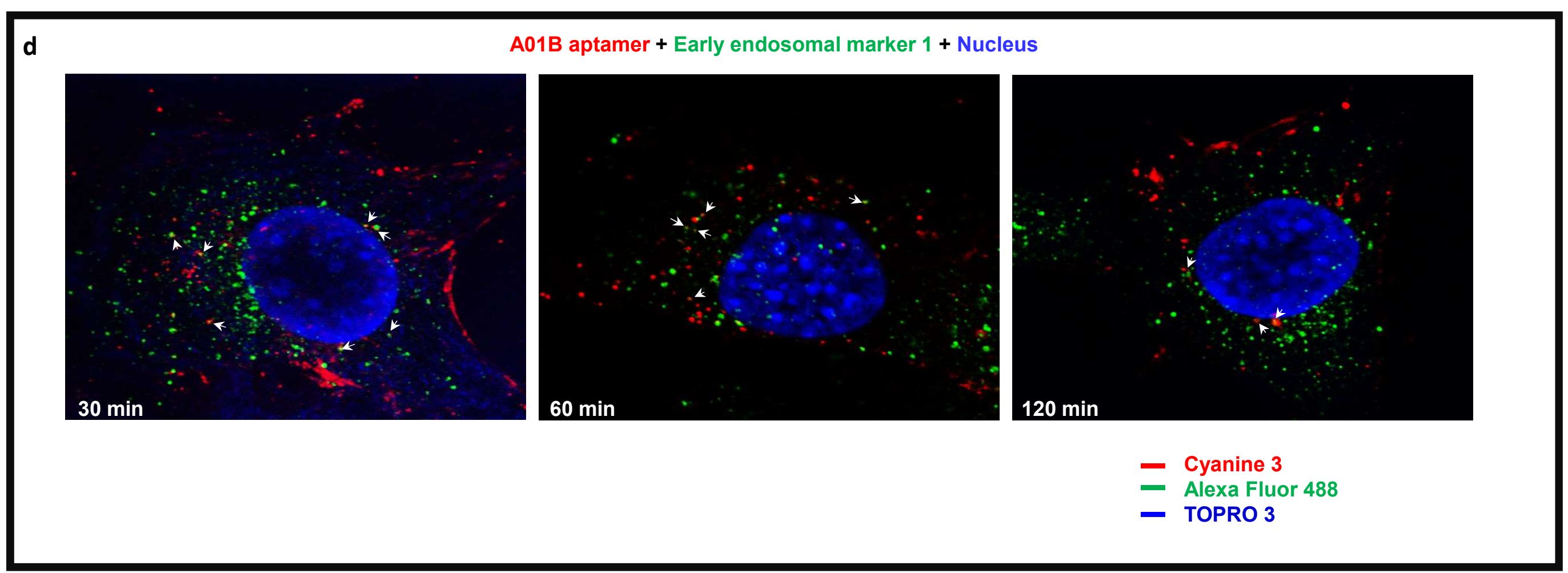




\section{A01B RNA aptamer}

\section{internalizes efficiently into skeletal muscle}

a

Cyanine 3
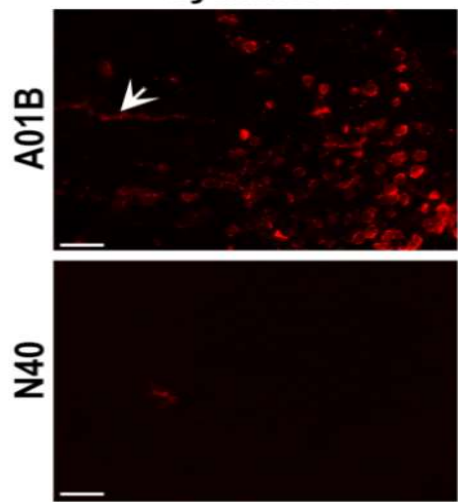

b
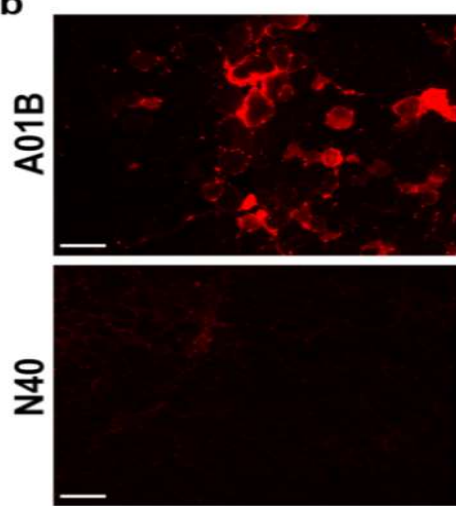

훙
Hoechst 33342
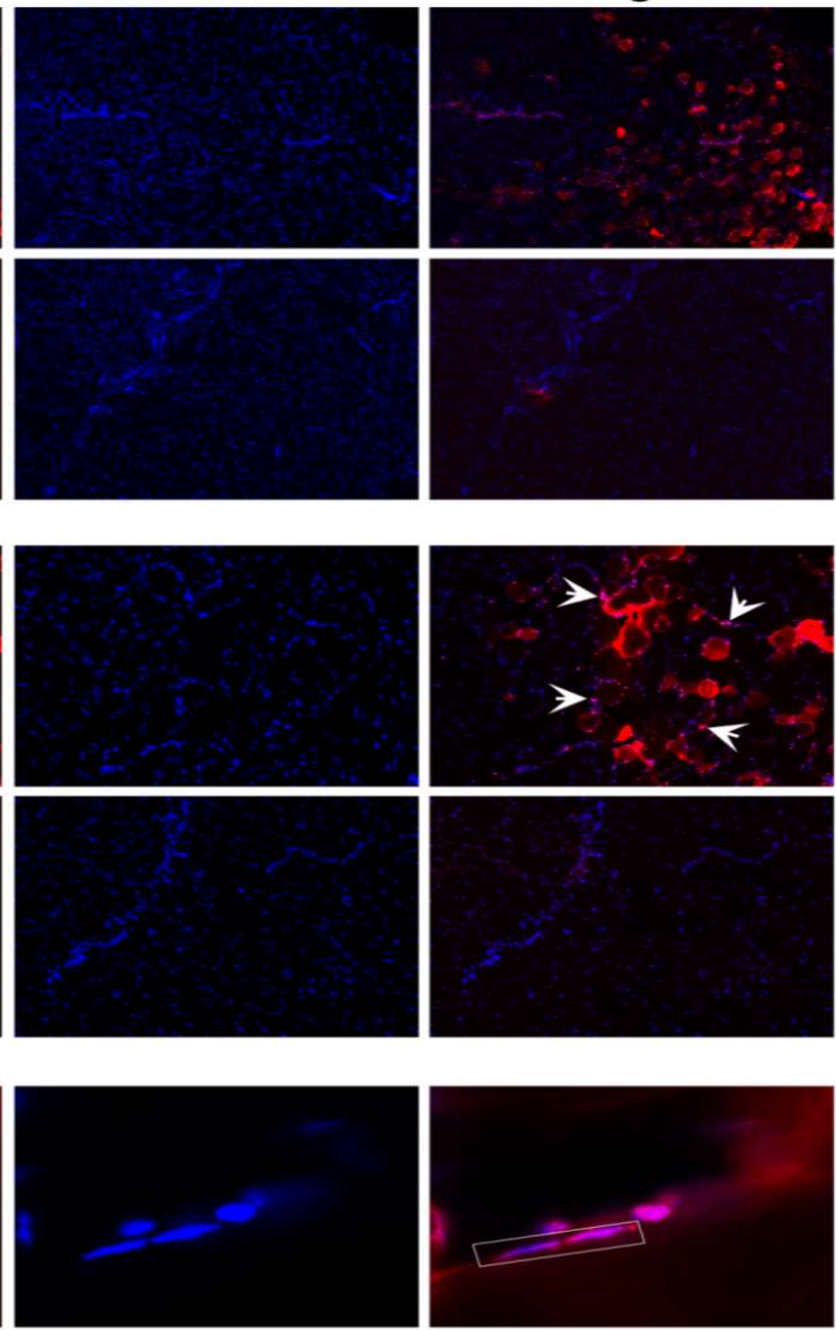

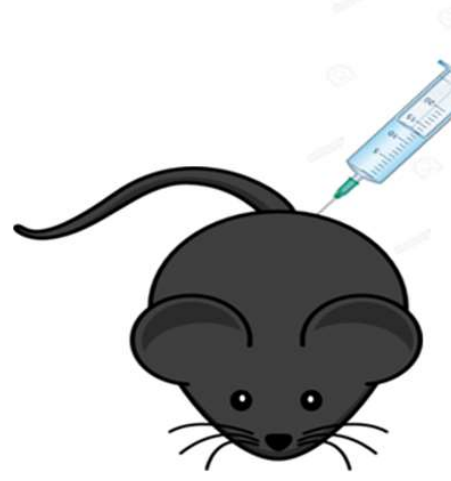

- Intramuscular injection of 100nM A01B RNA aptamer in TA muscle. Mice were sacrificed $1 \mathrm{~h}$ post injection

d

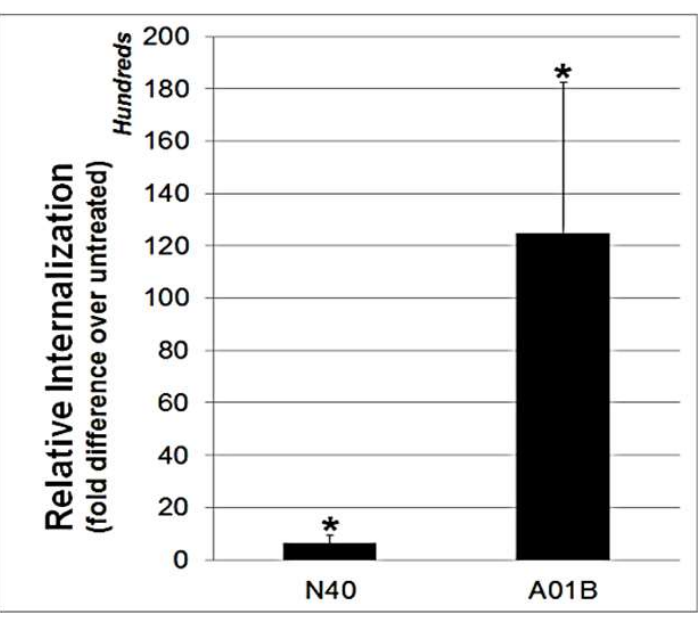




\section{Developing heart aptamers for AON delivery in DMD}




\section{Conclusions \\ \& FUTURE DIRECTIONS}

- Aptamers a novel approach to deliver specifically and efficiently to muscle

- First such aptamer discovered

- Incorporation of therapeutic oligonucleotides

-Specific targeting the heart muscle 


\section{Acknowledgements}

THE CYPRUS INSTITUTE OF NEUROLOGY \& GENETICS

* Department of Molecular Genetics, Function \& Therapy

- Melina Christou

- Constantina Costi

- Pavlos Fanis

- Kristia Georgiou

- Demetris Koutalianos

- Andrie Koutsoulidou

- Nikolas Mastroyiannopoulos

- Stalo Mytidou

- Vassos Neocleous

- Styliana Philippou

- Leonidas Phylactou

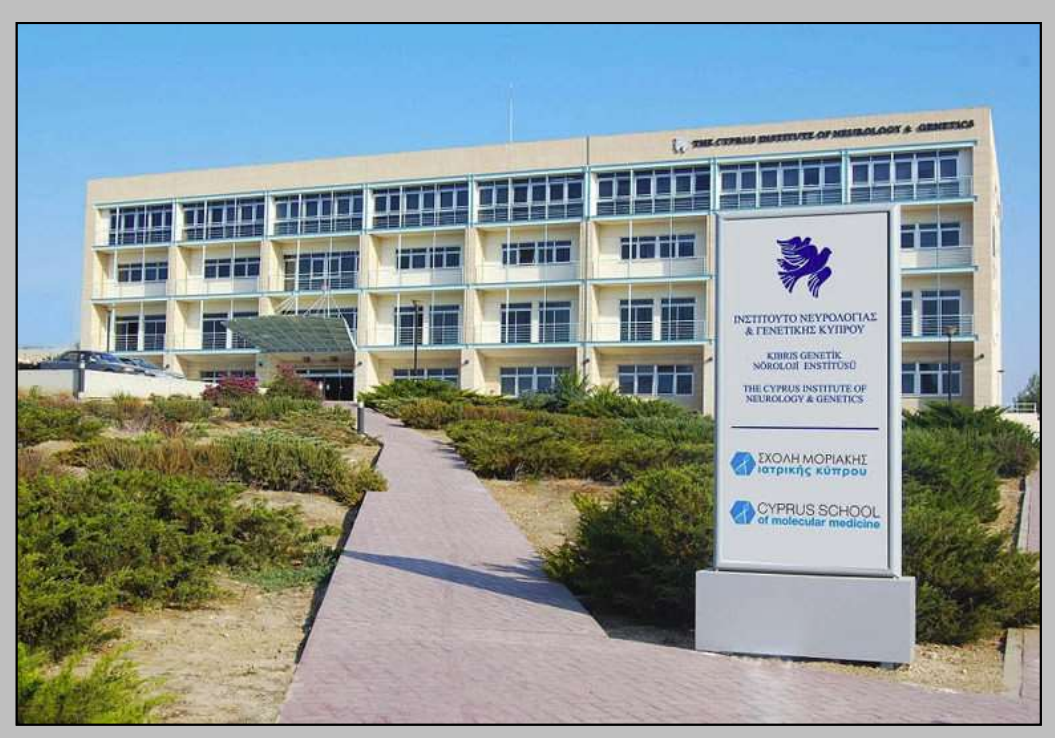

\section{FUNDING BODIES}

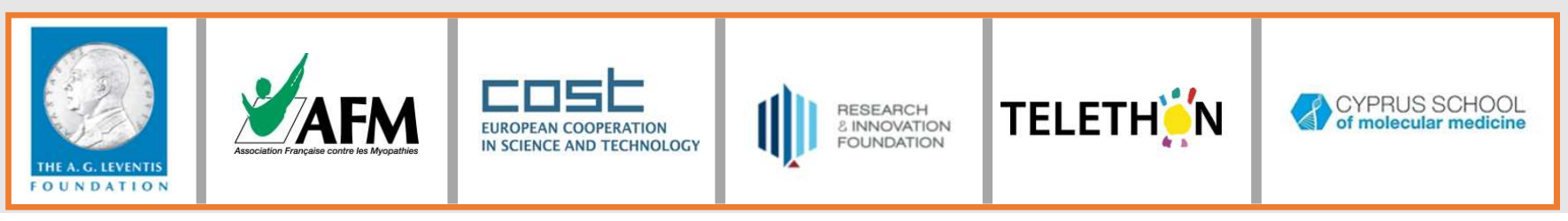

